In addition to the Lieb lattice, Drost et al. realize the $\mathrm{Su}-\mathrm{Schrieffer-}$ Heeger (SSH) model $^{9}$, the simplest of the topological models known in condensed matter. Initially, the SSH model was developed to account for solitons in polyacetylene ${ }^{9}$. And although the first simulation of the SSH model without polyacetylene was obtained in the framework of one-dimensional optical lattices ${ }^{10}$, the results presented by Drost et al. represent the first fully condensed-matter simulation of the model.

In the work by Slot et al. ${ }^{4}$, the Lieb lattice is achieved not by vacancies but through confinement of electronic surface states - a technique that is famously known for the quantum corral ${ }^{11}$, which was employed more recently to build so-called molecular graphene ${ }^{12}$. Here, carbon monoxide (CO) molecules placed on top of a $\mathrm{Cu}(111)$ surface locally deplete the two-dimensional electron gas that exists in the surface underneath. For this reason, to create the Lieb lattice the molecules are arranged to form a negative of the lattice (Fig. 1c). As such, this approach closely resembles the 'nearly free electron model', which is the opposite of the tight-binding approximation.
Although the Lieb lattice does not have an exact negative lattice (in the way that, for example, the triangular and honeycomb lattice are the exact negatives of each other ${ }^{12}$ ), Slot et al. obtained an optimal geometrical configuration of the CO molecules so as to resemble a suitable electrostatic potential profile as close as possible to the Lieb lattice. The complexity of this geometrical configuration is further increased by the different symmetry of the $\mathrm{Cu}(111)$ substrate and the Lieb lattice: triangular and square, respectively.

The experiments presented here by two independent groups demonstrate the viability of using atomically constructed lattices for quantum simulation. Many possibilities for future research can be envisioned, two of which we mention here. First, when the coherence length is long enough, complex junctions will make it possible to observe super-Klein tunnelling and super-collimation, which has already been predicted for this type of system ${ }^{13,14}$. Second, the use of a superconducting host material instead of a metal substrate could pave the way to study lattice systems of Cooper pairs. In this way, Feynman's vision could be accomplished entirely in condensed matter.
Dario Bercioux is in the Donostia International Physics Center (DIPC), Manuel de Lardizbal 4, E-20018 San Sebastian, Spain, and IKERBASQUE, Basque Foundation for Science, Maria Diaz de Haro 3, 48013 Bilbao, Spain. Sander Otte is in the Department of Quantum Nanoscience, Kavli Institute of Nanoscience, Delft University of Technology, Lorentzweg 1, 2628 CJ Delft, the Netherlands.

e-mail:dario.bercioux@dipc.org; a.f.otte@tudelft.nl

References

1. Feynman, R. P. Int. J. Theor. Phys. 21, 467-488 (1982)

2. Greiner, M. \& Folling, S. Nature 453, 736-738 (2008).

3. Drost, R., Ojanen, T., Harju, A. \& Liljeroth, P. Nat. Phys. 13, 669-672 (2017).

4. Slot, M. R. et al. Nat. Phys. 13, 673-677 (2017).

5. Vicencio, R. et al. Phys. Rev. Lett. 114, 245503 (2015)

6. Mukherjee, S. et al. Phys. Rev. Lett. 114, 245504 (2015)

7. Bradlyn, B. et al. Science 355, 5037 (2016).

8. Kalff, F. E. et al. Nat. Nanotech. 11, 926-929 (2016).

9. Su, W. P., Schrieffer, J. R. \& Heeger, A. J. Phys. Rev. Lett. 42, 1698-1701 (1979).

10. Atala, M. et al. Nat. Phys. 9, 795-800 (2013).

11. Crommie, M. F., Lutz, C. P. \& Eigler, D. M. Science 262, 218-220 (1993).

12. Gomes, K. K., Mar, W., Ko, W., Guinea, F. \& Manoharan, H. C. Nature 483, 306-310 (2012).

13. Urban, D. F., Bercioux, D., Wimmer, M. \& Häusler, W. Phys. Rev. B 84, 115136 (2011).

14. Fang, A., Zhang, Z. Q., Louie, S. G. \& Chan, C. T. Phys. Rev. B 93, 035422 (2016)

Published online: 24 April 2017

\title{
MAGNETIC SKYRMIONS
}

\section{On a wedge}

In certain magnetic materials, atomic spins can collectively order into vortex-like patterns known as magnetic skyrmions. Such skyrmions are highly mobile and have a very small (typically below $100 \mathrm{~nm}$ ) spatial extension - properties that have caught the attention of scientists developing a new data storage concept known as racetrack memory. In this type of non-volatile memory, skyrmions act as data carriers, capable of rapidly moving along a ferromagnetic strip for read/write operations. Clearly, a high degree of control of magnetic skyrmions is then required, and in this context, Chiming Jun and colleagues have now investigated the conditions needed for creating and manipulating skyrmions in nanoscale confinement (Nature Communications 8, 15569; 2017).

By means of ion-beam lithography, the authors first fabricated a nanosized wedge from a crystal of iron germanide (FeGe), with a width varying between about 10 and $180 \mathrm{~nm}$. Combining electron holography and image-reconstruction techniques, they were then able to obtain direct-space magnetic induction maps of the sample for different temperatures and external magnetic fields - providing the data for a phase diagram with magnetic field, wedge width and temperature as the phase variables. After cooling from room temperature to $220 \mathrm{~K}$, for example, an external field of about $150 \mathrm{mT}$ resulted in a single linear skyrmion chain (pictured).

The magnetic phase diagram also featured states like distorted helical spirals, pure edge twists and zigzag skyrmion chains. The variety of skyrmion phases seen in the FeGe nanowedge is absent in bulk and thin-film settings, as is the ability of the skyrmions to take on different sizes and ellipticities.

Jun et al. also presented a theoretical framework - based on evaluations of the energies of the skyrmion states - for numerical simulations of the observed phases. After checking that the calculated phases matched the experimentally observed situations, they numerically explored phase variable ranges not covered by the experimental phase diagram. Specifically, the authors looked at how elliptical skyrmions and their nucleation process develop, corroborating a conservation law for skyrmion numbers.

\section{BART VERBERCK}

